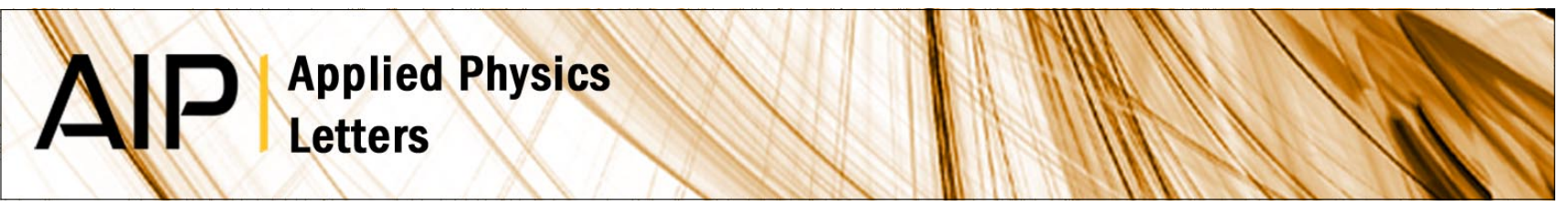

\title{
Collimation of horizontally polarized shear waves by means of ridge grating supported Love modes
}

\author{
J. Christensen, L. Martín-Moreno, and F. J. García-Vidal
}

Citation: Appl. Phys. Lett. 96, 233505 (2010); doi: 10.1063/1.3447929

View online: http://dx.doi.org/10.1063/1.3447929

View Table of Contents: http://apl.aip.org/resource/1/APPLAB/v96/i23

Published by the American Institute of Physics.

\section{Related Articles}

Far-field subwavelength imaging for ultrasonic elastic waves in a plate using an elastic hyperlens Appl. Phys. Lett. 98, 241912 (2011)

Actuators for the generation of highly nonlinear solitary waves

Rev. Sci. Instrum. 82, 034902 (2011)

Resonant slow modes in phononic crystal plates with periodic membranes

Appl. Phys. Lett. 97, 031902 (2010)

Rayleigh waves in air saturated axisymmetrical soft porous media

J. Appl. Phys. 106, 014906 (2009)

Achieving control of in-plane elastic waves

Appl. Phys. Lett. 94, 061903 (2009)

\section{Additional information on Appl. Phys. Lett.}

Journal Homepage: http://apl.aip.org/

Journal Information: http://apl.aip.org/about/about_the_journal

Top downloads: http://apl.aip.org/features/most_downloaded

Information for Authors: http://apl.aip.org/authors

\section{ADVERTISEMENT}

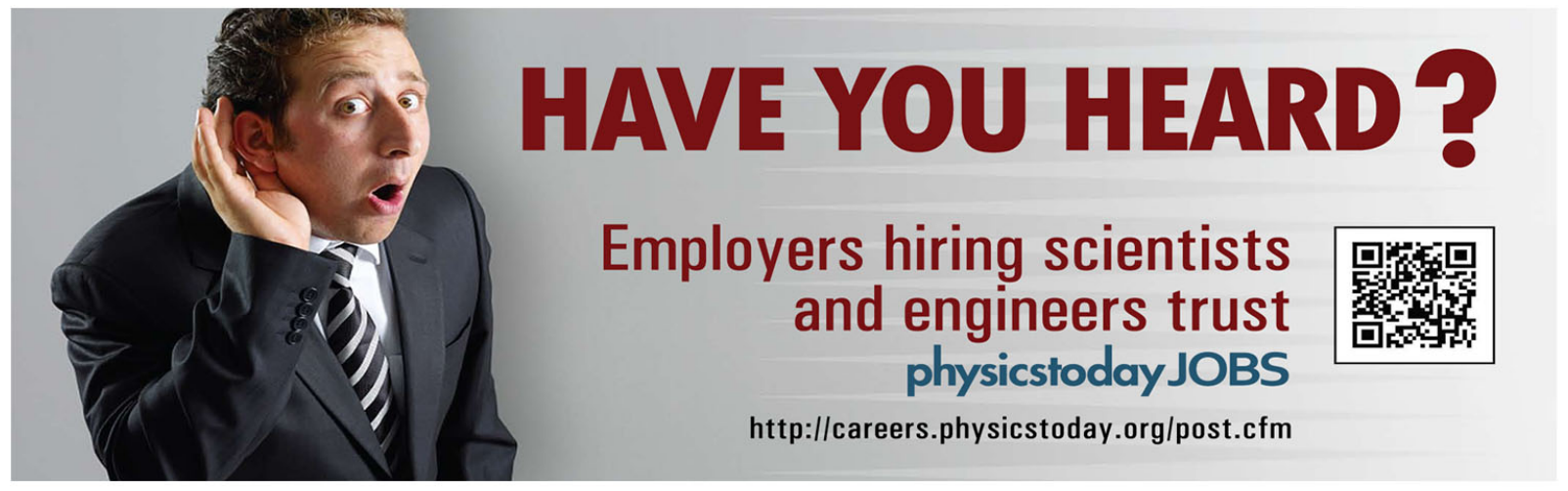




\title{
Collimation of horizontally polarized shear waves by means of ridge grating supported Love modes
}

\author{
J. Christensen, ${ }^{1, a)}$ L. Martín-Moreno, ${ }^{2}$ and F. J. García-Vidal ${ }^{1}$ \\ ${ }^{1}$ Departamento de Física Teórica de la Materia Condensada, Universidad Autónoma de Madrid, \\ E-28049 Madrid, Spain \\ ${ }^{2}$ Departamento de Física de la Materia Condensada and Instituto de Ciencia de Materiales de Aragón \\ (ICMA), CSIC-Universidad de Zaragoza, E-50009 Zaragoza, Spain
}

(Received 15 February 2010; accepted 16 May 2010; published online 7 June 2010)

\begin{abstract}
In this paper a theoretical study on transmission phenomena of horizontally polarized shear waves propagating through a subwavelength solid probe surrounded by ridges is presented. In this context, the resonant components responsible for enhanced transmission and elastic beaming are identified as Fabry-Perot resonances inside the probe, and ridge induced Love waves. Apart from the fundamental interest, it is envisaged that the ability to enhance elastic vibrations through subwavelength channels and to focus the emerged field, will give rise to improved ultrasonic devices for sensing and testing applications. (C) 2010 American Institute of Physics. [doi:10.1063/1.3447929]
\end{abstract}

The enhanced transmission of airborne sound through subwavelength apertures has attracted considerable interest both for isolated apertures ${ }^{1-3}$ such as one-dimensional ${ }^{4}$ and two-dimensional arrays. ${ }^{2,5,6}$ Transmission resonances through single slits or holes are governed by the plate thickness into which those apertures are milled and associated to the excitation of Fabry-Perot modes. Similar to surface plasmon polaritons responsible for enhanced transmission of light through perforated metallic films, ${ }^{7,8}$ acoustic surface waves play an important role when sound is funneled through narrow arrays of holes or slits. In this paper, we show how both enhanced transmission and beaming phenomena previously reported for electromagnetic waves impinging at single apertures surrounded by corrugations, ${ }^{9-11}$ also emerge for elastic waves. A theoretical prediction is presented for the first time where elastic waves are transmitted through a narrow subwavelength channel by means of surface modes that are similar to Love waves. ${ }^{12}$ Those Love waves that are supported by narrow surface ridges are not only giving rise to the enhanced transmission of elastic vibrations but also evoke the formation of a collimated beam. Since the late 1970s it was known that corrugated surfaces can support the existence of surface elastic waves. ${ }^{13-16}$ In particular, shear horizontal $(\mathrm{SH})$ polarized modes which do exist on substrates or metal samples when ridges are milled, grown or adsorbed onto them are widely used in connection with viscosity, gas, and (bio)chemical sensing such as nondestructive testing. ${ }^{17-19}$

The wave motion of a nonpiezoelectric, isotropic elastic material is governed by the general lossless equation of motion:

$$
\rho \frac{\partial^{2} u_{i}}{\partial t^{2}}=\frac{\partial \sigma_{i j}}{\partial x_{j}}
$$

with solid material mass density $\rho$, the stress $\sigma_{i j}$, and strain $\varepsilon_{i j}$ tensors, comprising Cartesian components $i, j=x, y, z$, given as follows:

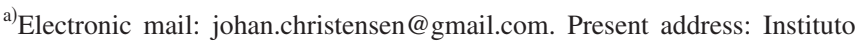
de Optica-CSIC Serrano 121, 28006 Madrid, Spain.
}

$$
\sigma_{i j}=\lambda \delta_{i j} \varepsilon_{k k}+2 \mu \varepsilon_{i j}, \quad \varepsilon_{i j}=\frac{1}{2}\left(\frac{\partial u_{i}}{\partial x_{j}}+\frac{\partial u_{j}}{\partial x_{i}}\right) .
$$

$\lambda, \mu$, and $u_{i}$ are the modulus of incompressibility (first Lame coefficient), modulus of rigidity (second Lame coefficient), and the $i$ th component of the displacement $u$, respectively. This given, one obtains the complete elastic wave equation comprising longitudinal (compressional) and transversal (shear) motion which for the sake of simplicity is given in vector notation

$$
\rho \frac{\partial^{2} \mathbf{u}}{\partial t^{2}}=(\lambda+2 \mu) \boldsymbol{\nabla}(\boldsymbol{\nabla} \cdot \mathbf{u})-\mu \boldsymbol{\nabla} \times \boldsymbol{\nabla} \times \mathbf{u} .
$$

In the following, two solid half-spaces are separated by a fluid layer while being interconnected by a probe as seen in the inset of Fig. 1. If a SH elastic wave is incident (along positive $z$-axis) on the stress free surface patterned by subwavelength ridges surrounding a narrow probe, we apply the curl $(\boldsymbol{\nabla} \times \mathbf{u})$ on the displacement vector in Eq. (3) and all

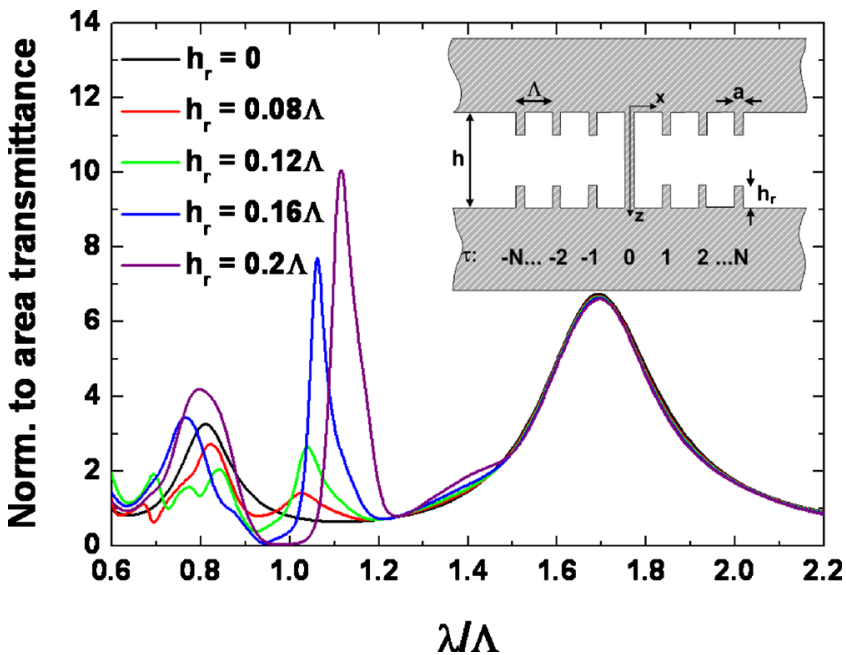

FIG. 1. (Color online) Influence on the normalized to area transmittance spectra at normal incidence radiation, dependent on the ridge height $h_{r}$ with $a=0.08 \Lambda, h=0.7 \Lambda$, and $N=5$. As the inset depicts, all ridges of same geometries are symmetrically placed around the probe. 
longitudinal terms vanish, which yields a wave equation

$$
\frac{\partial^{2} \mathbf{u}_{t}}{\partial t^{2}}=\frac{\mu}{\rho} \nabla^{2} \mathbf{u}_{t},
$$

with phase velocity $c_{t}=\sqrt{\mu / \rho}$ and transverse displacement vector $\mathbf{u}_{t}=\left(0, u_{y}, 0\right)$. The ridges are of width $a$ and height $h_{r}$, and are separated by $\Lambda$ whereas the probe has width $a$ and length $h$. The aim is efficiently to funnel shear polarized waves between two elastic half-spaces through a narrow subwavelength probe (aperture), $\lambda>\Lambda \gg a$. It is known that $\mathrm{SH}$ waves can satisfy shear-stress-free boundary conditions at all free interfaces: $\sigma_{x y}=\mu\left(\partial u_{y} / \partial x\right)=0$ at the ridge and probe faces such as $\sigma_{z y}=\mu\left(\partial u_{y} / \partial z\right)=0$ at the ridge ends and the facing half-spaces. The incident SH displacement $u_{y}$ reads

$$
\left|u_{y}^{i n c}(x, z)\right\rangle=\left|k_{x}^{0}\right\rangle e^{i k_{z}^{0} z}+\sum_{\gamma=-\infty}^{\infty} R_{\gamma}\left|k_{x}^{\gamma}\right\rangle e^{-i k_{z}^{\gamma}},
$$

with reflection coefficients $R_{\gamma}$, plane waves $\left\langle x \mid k_{x}^{\gamma}\right\rangle$ $=e^{i k_{x}^{\gamma} x} / \sqrt{\Lambda}, k_{x}^{\gamma}=k_{x}^{0}+(2 \pi / \Lambda) \gamma$ containing discrete diffraction order $\gamma$ in the range $\gamma=-\infty, \ldots 0 \ldots, \infty$ with $(2 \pi / \Lambda) \gamma$ representing the reciprocal lattice vector in the primitive cell of constant $\Lambda$. The ridges and the probe can be described in terms of their cavity modes: $\langle x \mid \tau, m\rangle$ $=\sqrt{\left(2-\delta_{0 m} / a\right)} \cos q_{x}^{m}\left(x-x_{\tau}+(a / 2)\right)$ if $\left|x-x_{\tau}\right|<(a / 2)$ otherwise zero, where $x_{\tau}=\tau \Lambda$ is the phase depicting the discrete ridge $(\tau)$ locations whereas the $m^{\text {th }}$ ridge/probe waveguide mode is in the range $(m=0,1,2 \ldots, \infty)$ with $q_{x}^{m}=m \pi / a$. The entire eigenvalue expression reads

$$
\left|u_{y}^{c a v}(x, z)\right\rangle=\sum_{m, \tau}\left(A_{m, \tau} e^{i q_{z}^{m} z}-B_{m, \tau} e^{-i q_{z}^{m} z}\right)|m, \tau\rangle,
$$

with $q_{z}^{m}=k_{0} \sqrt{n^{2}-(m \lambda / 2 a)^{2}}$. When the wave emerges the probe, the fields are expanded out in linear diffracted Bloch waves with $T_{\gamma}$ being the transmission coefficient

$$
\left|u_{y}^{e m g}(x, z)\right\rangle=\sum_{\gamma=-\infty}^{\infty} T_{\gamma}\left|k_{x}^{\gamma}\right\rangle e^{i k_{z}^{\gamma}(z-h)} .
$$

Within the modal expansion technique, continuity along all interfaces is preserved for $u_{y}$ and $\sigma_{z y}$ whereupon Eqs. (5)-(7) can be assembled into a linear system:

$$
\begin{gathered}
\left(G_{\tau \tau}-\epsilon\right) \sigma_{\tau}+\sum_{\tau^{\prime} \neq \tau} G_{\tau \tau^{\prime}} \sigma_{\tau^{\prime}}-\delta_{\tau 0} G^{V} \sigma_{0}^{\prime}=I_{\tau}^{0}, \\
\left(G_{\tau \tau}-\epsilon\right) \sigma_{\tau}^{\prime}+\sum_{\tau^{\prime} \neq \tau} G_{\tau \tau^{\prime}} \sigma_{\tau^{\prime}}^{\prime}-\delta_{\tau 0} G^{V} \sigma_{0}=0 .
\end{gathered}
$$

This system gathers the entire elastic wave problem under one umbrella in which the modal stress components $\sigma_{\tau}, \sigma_{\tau}^{\prime}$ and all other expansion coefficients can be solved. As of a subwavelength probe and ridges, we assumed that only the fundamental cavity mode is excited, which, e.g., simplifies the function coupling ridges $\tau$ with $\tau^{\prime}$ over diffracted $\mathrm{SH}$ waves by means of a Green's function containing $H_{0}^{(1)}$, the zero-order Hankel function of the first kind

$$
G_{\tau \tau^{\prime}}=\frac{i \pi}{\lambda} \int_{x_{\tau^{-}-a / 2}}^{x_{\tau^{+a / 2}}} \int_{x_{\tau^{\prime}-a / 2}}^{x_{\tau^{\prime}}+a / 2} H_{0}^{(1)}\left(k_{0}\left|x-x^{\prime}\right|\right) d x d x^{\prime} .
$$

Note also how this function has been reformulated into a continuous diffraction problem. All other functions in Eq. (8) are similar to the ones given in Ref. 2. In Fig. 1 we study the influence of the ridge height $h_{r}$ on the transmittance normalized to the probe width, $T_{\text {probe }}=1 / a \operatorname{Im}\left(\Sigma_{\tau} \delta_{\tau 0} G^{V} \sigma_{\tau} \sigma_{\tau}^{\prime *}\right)$, at normal incidence. Transmittance through the probe in the absence of ridges $h_{r}=0$ is dominated by two peaks that are only affected weakly when the ridge height is more pronounced (upto $h_{r}=0.2 \Lambda$ ). These are the two first Fabry-Perot resonances $\lambda_{l}=2 h / l$ of order $l$ which stem from SH standing waves inside the probe. However, with the introduction of ridges, we are able to excite leaky Love waves that are giving rise to the enhanced transmission at a wavelength close to the period $\lambda \gtrsim \Lambda$ seen in Fig. 1. In order to get insight into those ridge induced Love modes, we study the divergence of the reflection coefficient $R_{\gamma}$ from a ridge-grating, which easily is applied from our system if the probe is disregarded and only the first half-space is considered. Specular reflection dominates the process if $\lambda \gg \Lambda$ where higher diffraction order safely can be neglected, hence

$$
R_{0}=\frac{1+i \frac{k_{0}}{k_{z}} \frac{a}{\Lambda} \tan k_{0} h_{r}}{1-i \frac{k_{0}}{k_{z}} \frac{a}{\Lambda} \tan k_{0} h_{r}},
$$

that for evanescent ridge-states is determining the following dispersion relation:

$$
k_{x}=k_{0} \sqrt{1+\frac{a^{2}}{\Lambda^{2}} \tan ^{2} k_{0} h_{r}} .
$$

Similar to the plasma frequency of a free electron gas in metals, Eq. (11) approaches $\omega_{\text {Love }}=\pi c / 2 h_{r}$ for infinite parallel momentum. It can conclusively be seen that resonant transmission of sound in periodic structures is aided by the excitation of localized Fabry-Perot modes and ridge surface resonances. Moreover, in a more generalized context we can write the requirement for a Fabry-Perot mode as $\sin n k_{0} h$ $=0$ where $n=\rho_{r} / \mu_{r}$ is the index of refraction of the probe relative to the surrounding. Instead of tuning the structures geometrical parameter in order to exhibit maximum transmission resonances, we are shifting the location of the Fabry-Perot modes by a probe material (characterized by $n$ ) different from the surrounding half-spaces, $\lambda_{l}=n(2 h / l)$, ideally into the spectral location of the ridge induced Love modes. Concerning this matter, Fig. 2 is illustrating how this leads to a strong coupling of those modes giving rise to increased transmission enhancement, relative to the probe width. Bearing in mind that the wavelength scales with the SH velocity $c_{t}$ inside the probe, hence an increasing index of refraction leads to multiple resonances as of the reduced wavelength inside the probe.

The structural parameters chosen do not only lead to enhanced transmission of $\mathrm{SH}$ waves through the subwavelength probe but yield the formation of a collimated displacement $|\mathbf{u}|$ beam in the farfield. When vibrations are emerging the probe, ridge Love modes are also excited at that interface which by means of constructive interference is giving rise to an elongated focus in the farfield. Figure 3 is illustrating this 


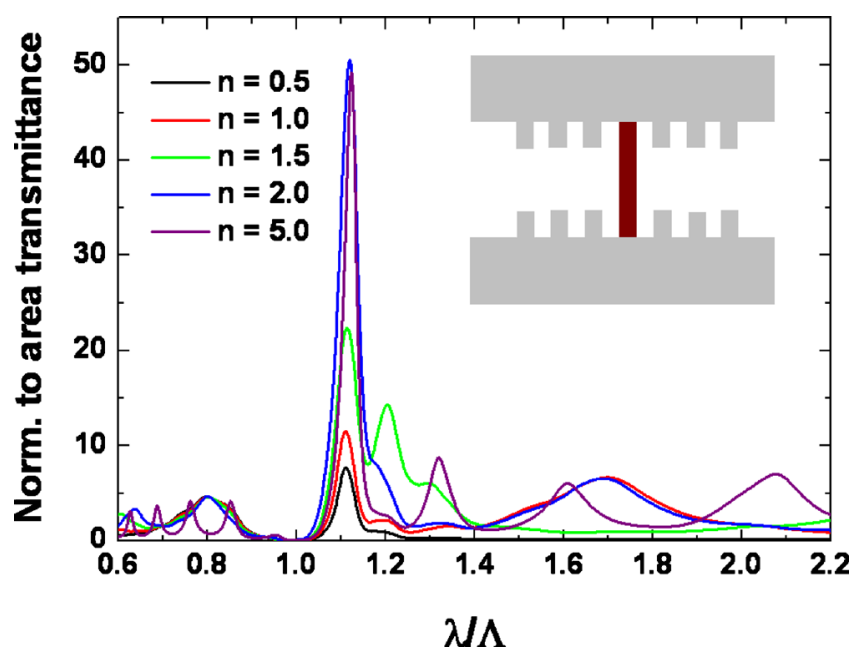

FIG. 2. (Color online) Influence of the probe index of refraction $n$ on the normalized to area transmittance spectra at normal incidence, for a structure with $a=0.08 \Lambda, h=0.7 \Lambda, h_{r}=0.2 \Lambda$, and $N=5$.

for a system (see caption) containing different probes regarding the index of refraction $n$. Even though transmission greatly is enhanced with increased $n$ due to the coupling of a Fabry-Perot resonance with ridge Love modes, the time averaged displacement distribution $|\mathbf{u}|$ is only slightly raised [Fig. 3(b)] compared to the case of $n=1$ inside the probe [Fig. 3(a)].

To control the flow of vibrational wave motion on a subwavelength scale, seems to unify the classical picture on

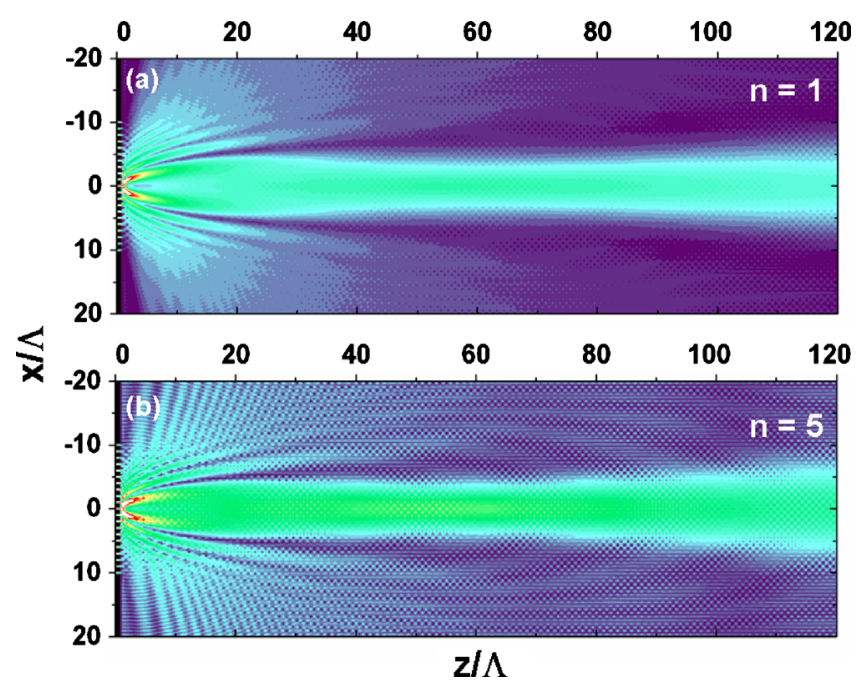

FIG. 3. (Color online) Farfield $(z>0)$ map of the displacement field $|\mathbf{u}|$ for a configuration with the geometries: $a=0.08 \Lambda, h=0.7 \Lambda, h_{r}=0.2 \Lambda$, and $N=10$. (a) Farfield plot for $n=1$ at $\lambda \approx 1.1 \Lambda$. (b) Farfield plot for $n=5$ at $\lambda \approx 1.15 \Lambda$. the extraordinary transmission of waves through small apertures for light, sound, matter, and elastic waves. ${ }^{20}$ Those $\mathrm{SH}$ waves described here are different from longitudinal airborne sound, however would not add additional rigour in an experimental implementation. All geometries are normalized to the lattice constant $\Lambda$, which means that the structure determining the resonant frequencies can be scaled at will. The ability to concentrate horizontally polarized shear waves on a scale smaller than the wavelength and efficiently to squeeze them through a narrow channel followed by an elongated focus, opens up exciting possibilities to improve ultrasonic scanning for detecting small and deep hidden defects in materials caused by fatigue.

This work was financially supported by the Spanish Ministry of Science and Innovation under Project Nos. MAT2008-06609-C02 and CSD2007-046-Nanolight.es. J.C. gratefully acknowledges financial support from the Carlsberg Foundation under Contract No. QUANTONICS 2009-010167.

${ }^{1}$ J. Christensen, A. I. Fernandez-Dominguez, F. de Leon-Perez, L. MartinMoreno, and F. J. Garcia-Vidal, Nat. Phys. 3, 851 (2007),

${ }^{2}$ J. Christensen, L. Martin-Moreno, and F. J. Garcia-Vidal, Phys. Rev. Lett. 101, 014301 (2008).

${ }^{3}$ J. Mei, B. Hou, M. Ke, S. Peng, H. Jia, Z. Liu, J. Shi, W. Wen, and P. Sheng, Appl. Phys. Lett. 92, 124106 (2008).

${ }^{4}$ M. H. Lu, X. K. Liu, L. Feng, J. Li, C. P. Huang, and Y. F. Chen, Phys. Rev. Lett. 99, 174301 (2007)

${ }^{5}$ B. Hou, J. Mei, M. Z. Ke, J. W. Wen, Z. Y. Liu, J. Shi, and P. Sheng, Phys. Rev. B 76, 054303 (2007).

${ }^{6}$ H. Estrada, P. Candelas, A. Uris, F. Belmar, F. J. G. de Abajo, and F. Meseguer, Phys. Rev. Lett. 101, 084302 (2008).

${ }^{7}$ T. W. Ebbesen, H. J. Lezec, H. F. Ghaemi, T. Thio, and P. A. Wolff, Nature (London) 391, 667 (1998).

${ }^{8}$ J. B. Pendry, L. Martin-Moreno, and F. J. Garcia-Vidal, Science 305, 847 (2004).

${ }^{9}$ H. J. Lezec, A. Degiron, E. Deveux, R. A. Linke, L. Martin-Moreno, F. J. Garcia-Vidal, and T. W. Ebbesen, Science 297, 820 (2002).

${ }^{10}$ L. Martín-Moreno, F. J. Garcia-Vidal, H. J. Lezec, A. Degiron, and T. W. Ebbesen, Phys. Rev. Lett. 90, 167401 (2003).

${ }^{11}$ F. J. García-Vidal, H. J. Lezec, T. W. Ebbesen, and L. Martin-Moreno, Phys. Rev. Lett. 90, 213901 (2003).

${ }^{12}$ A. E. H. Love, Some Problems of Geodynamics (Cambridge University Press, Cambridge, 1911).

${ }^{13}$ B. A. Auld, J. J. Gagnepain, and M. Tan, Electron. Lett. 12, 650 (1976)

${ }^{14}$ Y. V. Gulyaev and V. P. Plesskii, Sov. Tech. Phys. Lett. 3, 87 (1977).

${ }^{15}$ A. A. Maradudin and W. Zierau, Geophys. J. Int. 118, 325 (1994).

${ }^{16}$ A. Khelif, B. Djafari-Rouhani, and Ph. Lambin, Eur. Phys. J. B 21, 437 (2001).

${ }^{17}$ J. Du, G. L. Harding, J. A. Ogilvy, P. R. Dencher, and M. Lake, Sens. Actuators, A 56, 211 (1996).

${ }^{18}$ J. Xu, J. S. Thakur, F. Zhong, H. Ying, and G. W. Auner, J. Appl. Phys. 96, 212 (2004).

${ }^{19}$ M.-I. Rocha-Gaso, C. March-Iborra, A. Montoya-Baides, and A. ArnauVives, Sensors 9, 5740 (2009).

${ }^{20}$ A. I. Fernández-Dominguez, D. Martin-Cano, E. Moreno, L. MartinMoreno, and F. J. Garcia-Vidal, Phys. Rev. A 78, 023614 (2008). 